\title{
StellaTUM: current consensus and discussion on pancreatic stellate cell research
}

Mert Erkan, ${ }^{1}$ Guido Adler, ${ }^{2}$ Minoti V Apte, ${ }^{3}$ Max G Bachem, ${ }^{4}$ Malte Buchholz, ${ }^{5}$ Sönke Detlefsen, ${ }^{6}$ Irene Esposito, ${ }^{7}$ Helmut Friess, ${ }^{1}$ Thomas M Gress, ${ }^{5}$ Hans-Joerg Habisch, ${ }^{4}$ Rosa F Hwang, ${ }^{8}$ Robert Jaster, ${ }^{9}$ Jörg Kleeff, ${ }^{1}$ Günter Klöppel, ${ }^{7}$ Claus Kordes, ${ }^{10}$ Craig D Logsdon, ${ }^{8}$ Atsushi Masamune, ${ }^{11}$ Christoph W Michalski, ${ }^{1}$ Junseo $\mathrm{Oh}^{12}$ Phoebe A Phillips, ${ }^{3}$ Massimo Pinzani, ${ }^{13}$ Carolin Reiser-Erkan, ${ }^{1}$ Hidekazu Tsukamoto, ${ }^{14}$ Jeremy Wilson ${ }^{3}$

\begin{abstract}
The field of pancreatic stellate cell (PSC) biology is very young, as the essential invitro tools to study these cells (ie, methods to isolate and culture PSC) were only developed as recently as in 1998. Nonetheless, there has been an exponential increase in research output in this field over the past decade, with numerous research groups around the world focusing their energies
\end{abstract}

\footnotetext{
${ }^{1}$ Department of Surgery, Klinikum rechts der Isar, TU München, Munich, Germany; ${ }^{2}$ Department of Internal Medicine, Johannes Gutenberg-Universität Mainz, Mainz, Germany; ${ }^{3}$ Faculty of Medicine, The University of New South Wales, Sydney, New South Wales, Australia; ${ }^{4}$ Department of Clinical Chemistry, Universitätsklinikum Ulm, Ulm, Germany; ${ }^{5}$ Department of Internal Meidicine, Universitätsklinikum Marburg, Marburg, Germany; ${ }^{6}$ Department of Pathology, Odense University Hospital, Odense, Denmark; ${ }^{7}$ Institute of Pathology, Klinikum rechts der Isar, TU München, Munich, Germany; ${ }^{8}$ Department of Surgical Oncology, University of Texas MD Anderson Cancer Center, Houston, Texas, USA; ${ }^{9}$ Department of Internal Medicine University of Rostock, Rostock, Germany; ${ }^{10}$ Department of Gastroenterology, Hepatology and Infectious Diseases, Universitätsklinikum Düsseldorf, Düsseldorf, Germany; ${ }^{11}$ Division of Gastroenterology, Tohoku University Graduate School of Medicine, Sendai, Miyagi, Tohoku, Japan; ${ }^{12}$ Laboratory of Cellular Oncology, Korea University Graduate School of Medicine, Seoul, South Korea; ${ }^{13}$ Department of Internal Medicine, University of Florence, Florence, Italy; ${ }^{14}$ Southern California Research Center for ALPD and Cirrhosis, Keck School of Medicine of the University of Southern California, Los Angeles, California, USA
}

Correspondence to Dr Mert Erkan, Department of General Surgery, Technische Universität München, Klinikum rechts der Isar, 81675 Munich, Germany; m.mert.erkan@googlemail.com into elucidating the biology and function of these cells. It is now well established that PSC are responsible for producing the stromal reaction (fibrosis) of two major diseases of the pancreas-chronic pancreatitis and pancreatic cancer. Despite exponentially increasing data, the methods for studying PSC remain variable. Although within individual laboratories methods are consistent, different methodologies used by various research groups make it difficult to compare results and conclusions. This article is not a review article on the functions of PSC. Instead, members of the Pancreatic Star Alliance (http://www. pancreaticstaralliance.com) discuss here and consolidate current knowledge, to outline and delineate areas of consensus or otherwise (eg, with regard to methodological approaches) and, more importantly, to identify essential directions for future research.

\section{PANCREATIC STELLATE CELLS}

Hepatic stellate cells (HSC) were first described by Karl von Kupffer in 1876; however, similar cells in the pancreas were first observed in the 1980s. ${ }^{1-3}$ In 1998, Apte et $a l^{4}$ and Bachem et $a l^{5}$ isolated and cultured PSC. ${ }^{4}$ In the normal pancreas, PSC are located in close proximity to the basal aspect of pancreatic acinar cells. In sections immunostained for the marker desmin (a cytoskeletal protein), quiescent PSC can be seen as cells with a central cell body and long cytoplasmic projections extending along the base of adjacent acinar cells similar to that of pericytes in the mammary gland. In health, PSC exist in their quiescent phenotype and exhibit the presence of abundant vitamin A-containing lipid droplets in their cytoplasm. It is estimated that quiescent PSC form $4-7 \%$ of all parenchymal cells in the normal pancreas. ${ }^{4} 5$ During pancreatic injury, resident PSC transform into an activated phenotype that secretes excessive amounts of the extracellular matrix (ECM) proteins that comprise fibrous tissue. Recent evidence suggests that a small proportion of activated PSC may also be derived from circulating bone marrow (BM)-derived cells that home to the pancreas during pancreatic injury.

\section{STELLATE CELL SYSTEM AND THE ORIGIN OF PSC AND HSC}

In the human body, the stellate cell system consists of retinoid-storing cells in various organs, including the liver, pancreas, lung, kidney, intestine, spleen, adrenal gland, ductus deferens and vocal cords showing a perivascular location with a distribution typical of a pericyte. ${ }^{67}$ However, the origin of stellate cells is still being debated. Mesenchymal, ${ }^{8-10}$ endodermal $^{11} 12$ as well as neuroectodermal ${ }^{13-15}$ origins are suggested. Neuroectoderm and mesoderm were considered as two potential origins of HSC-these cells and PSC share numerous characteristics as indicated by morphological, functional and gene expression studies. Expression profiling of PSC, HSC and fibroblasts has demonstrated that PSC and HSC are distinctly different from fibroblasts, but share many homologies including the expression of genes related to ECM proteins, contractility, retinoid metabolism (although lower retinoid content in PSC compared with HSC) and growth factors. ${ }^{16}$ In fact, both cells (in comparison with fibroblasts) have more similarities than differences. ${ }^{17}$ It is therefore possible that HSC and PSC share a common origin.

The neuroectoderm proposal for the origins of HSC was refuted by a study by Cassiman et al, ${ }^{10}$ who used a genetic cell lineage mapping technique with Rosa26YFP flox mice crossed with mice expressing Cre under the control of the neural crest-specific Wnt1 promoter/ enhancer. Definitive novel evidence for the mesodermal origin of HSC has recently been presented by Asahina and colleagues $^{8} 18$ with the use of the mesoderm-specific MesP1Cre. A conditional cell lineage analysis using $\mathrm{Wt} 1^{\text {CreERT/ }}$ Rosa26Lac ${ }^{\text {flox }}$ or ROSA26mTmG flox mice, 
revealed Wt1-positive septum transversum giving rise to mesothelial cells, submesothelial cells, HSC and perivascular mesenchymal cells during liver development. ${ }^{8}$ That study also demonstrated Wt1-positive mesothelial/submesothelial cells migrating inward from the liver surface to generate liver mesenchymal cells including HSC. Similar lineage tracing techniques need to be used to determine the exact origin of PSC.

\section{ROLE OF PSC IN FIBROSIS AND REGENERATION}

The origin and fate of stellate cells in the context of injury and regeneration are also a matter of ongoing debate. Regarding the contribution of BM and epithelial mesenchymal transition (EMT) of acinar cells to the PSC population, the data are limited and restricted to studies in mice. In 2006, it was reported that BM is a source of myofibroblast-like cells in fibrotic liver tissue, but the involvement of these cells in the progression of liver fibrosis remains questionable as their contribution to collagen synthesis appears to be limited. ${ }^{19} 20$ Recent studies have now also addressed the role of BM-derived cells in pancreatic regeneration and fibrosis. ${ }^{21} 22$ Using a similar experimental approach, namely the transfer of green fluorescent protein (GFP)-expressing BM cells to lethally irradiated small rodents followed by the induction of chronic pancreatitis with cerulein or dibutyltin dichloride, it was consistently shown that BM-derived cells did home to the pancreas. ${ }^{21} 22$ In both studies, the induction of chronic pancreatitis was found to be associated with an increase in the number of GFP-expressing PSC. Even then, however, no more than $20 \%$ of all PSC expressed GFP, indicating a predominance of resident over BM-derived stellate cells. ${ }^{22}$ Together, these experimental data point to a significant but quantitatively limited contribution of BM to the pool of PSC. Although there are also some data that EMT of acini may contribute to the ultimate PSC population (M. Erkan et al, unpublished data), there is general agreement that there is not currently enough evidence to support the latter hypothesis. As of now, the situation in humans is not known. The effects of BMderived and EMT-derived PSC on the course of inflammation, repair and fibrosis of the pancreas remains to be further elucidated. ${ }^{23}$

Regarding hepatic and pancreatic regeneration after injury in rodents, there is some preliminary evidence for HSC and PSC acting as stem cells in respective organs. HSC of rats express markers such as nestin and CD133, which are known to be expressed on somatic stem cells. $^{24}$ However, before HSC can be classified as stem cells, essential characteristics of stem cells should be defined and verified in stellate cells. Briefly, stem cells are undifferentiated cells with the potential to proliferate and to undergo developmental processes. More specifically, stem cells: (1) express genes required for the inhibition or induction of cell development; (2) maintain their characteristics in a special microenvironment (stem cell niche); (3) are normally quiescent, but can be activated on demand; (4) proliferate/migrate when activated; and (5) can differentiate into effector cells (plasticity) or influence the developmental fate of other cells. Through these mechanisms stem cells participate in ontogenesis, regeneration or reproduction of organisms. Furthermore, stem cells are transplantable and can survive for a long time within host tissues. First steps were made to unravel these characteristics in HSC of rodents. Genes required for stemness and developmental processes are expressed by HSC, which preserve their quiescent state within their niche, the space of Dissé. ${ }^{24} 25$ Moreover, HSC are quiescent in normal liver, but become activated especially during stem cell-based liver repair. The gene expression of different cell types such as hepatocytes is inducible in activated HSC by cytokine treatment or co-culture with parenchymal cells, demonstrating their plasticity. ${ }^{24} 25$ In addition, some evidence exists that HSC can also contribute to liver repair. ${ }^{26}$ With regard to the pancreas, a recent study has described a subset of mitoxantrone (a type II topoisomerase inhibitor that disrupts DNA synthesis and DNA repair in both healthy cells and cancer cells) resistant pancreatic cells that exhibit PSC markers, and can be induced to differentiate into insulin producing $\beta$ cells when exposed to an appropriate culture medium. ${ }^{27}$ It is possible that PSC may also express various markers that are expressed in stem cells. However, convincing functional data showing that PSC may in fact transform into other cell types of the pancreas are not yet available. Whether PSC contribute to pancreas regeneration in a manner similar to the contribution of HSC to liver regeneration, by supporting the epithelial cells and by developing into epithelial cells, ${ }^{26}$ remains to be analysed. As of now, the situation in humans remains completely unknown.

\section{METHODOLOGY FOR ISOLATION OF PSC}

Isolation of quiescent PSC from rat pancreas was first reported by Apte and colleagues ${ }^{4}$ from Professor Wilson's laboratory in 1998. This method took advantage of the known vitamin A content (stored in cytoplasmic lipid droplets) of PSC, which allowed the cells to be separated by a single density gradient method using nycodenz. More recently, a similar method (albeit with some modifications) was used to isolate quiescent PSC from normal human pancreas. ${ }^{28}$ The gradients can be developed not only with nycodenz but also with other colloids such as percoll, iohexol, iodixanol, optiprep. The average yield of cells from a rat pancreas is approximately 3 million cells per gram of pancreas. The yield of PSC is much lower from human pancreas due to limited tissue availability and due to the higher amount of fatty tissue surrounding the resected pancreas (compared with rat pancreas), which can impede tissue digestion. PSC can also be isolated from cancerous or fibrotic human pancreas, as well as normal rat pancreas, using the outgrowth method first described by Bachem and colleagues ${ }^{5}$ from Professor Adler's laboratory in 1998. The PSC obtained by the outgrowth method comprise cells of different age due to temporal differences in their growing out from fibrotic tissue blocks. Both methods yield a mixed population of stromal cells, which are globally named PSC but have different subpopulations with various sizes, $\boldsymbol{\alpha}$-smooth muscle actin ( $\boldsymbol{\alpha}$-SMA) expression and adhesiveness on plastic. Neither method yields absolutely pure PSC populations, although the nycodenz method yields a more homogeneous population of PSC at the point of isolation than the outgrowth method, which is inherently prone to possible contamination by other cells. In early primary cultures obtained by outgrowth methods, the contaminating cells are predominantly macrophages and acinar cells. After passaging, the cultures may also contain $\alpha$-SMA-negative fibroblasts while acinar cells and macrophages are eliminated. Tumour cells almost never grow out from primary tumours, but may seldom grow out from metastasis samples. ${ }^{29}$ Nonetheless, good practice requires checking cell preparations for quality and purity regardless of the isolation method used.

\section{MARKERS OF QUIESCENT AND ACTIVATED PSC}

When isolating primary cells from the pancreas, it is of vital importance to assess the purity of the cultures not only for the homogeneity of PSC but also for the 
absence of possible contaminants. Another confounder is the activation of PSC on plastic, which creates differences between early and late passages. Therefore, several PSC selective markers have been proposed to assess their purity and activity (figure 1). The most consistent of vitamin A droplets in the cytoplasm. With regard to activated PSC, it is currently not clear whether these are different from myofibroblasts of the pancreas and whether there are markers to differentiate these two cell types. However, there is strong agreement that $\alpha$-SMA is not an exclusive marker for activated PSC. It is also expressed by myofibroblasts, smooth muscle cells and pericytes in blood vessels as well as the gut wall (ie, duodenum and papilla vateri). During activation, quiescent PSC lose their intracellular retinoid (vitamin A) droplets (detectable by fluorescence microscopy as a characteristic blue-green autofluorescence (rapidly fading) upon exposure to ultraviolet light at $328 \mathrm{~nm}$ or $350 \mathrm{~nm}$ ) and start to express $\alpha$-SMA. The active phenotype of PSC defined by $\alpha$-SMA expression may have contrasting marker of PSC quiescence is the presence

functions, eg, profibrogenic or profibrolytic, therefore $\alpha$-SMA is considered to be more a transdifferentiation marker indicative of a myofibroblast-like phenotype than a marker of PSC activity per se. ${ }^{30}$

With regard to other PSC markers, researchers are in agreement that the expression of desmin is highly variable in PSC (variance of expression also exists between different species). Similarly, there is currently not enough evidence to conclude that nestin alone is a sufficient marker to monitor the activation of PSC in rodents and humans. A more commonly used and reliable immunostaining marker selective for rat and human PSC (and absent in fibroblasts) is glial fibrillar acidic protein. Some researchers have reported the absence of glial fibrillar acidic protein expression in quiescent PSC, but in the opinion of experienced workers in the field this is more likely to be due to differences in the quality of the antibody used for immunostaining than a true absence of expression of the protein.

The dynamic change in gene expression during PSC activation can be monitored by several gene products. There is some

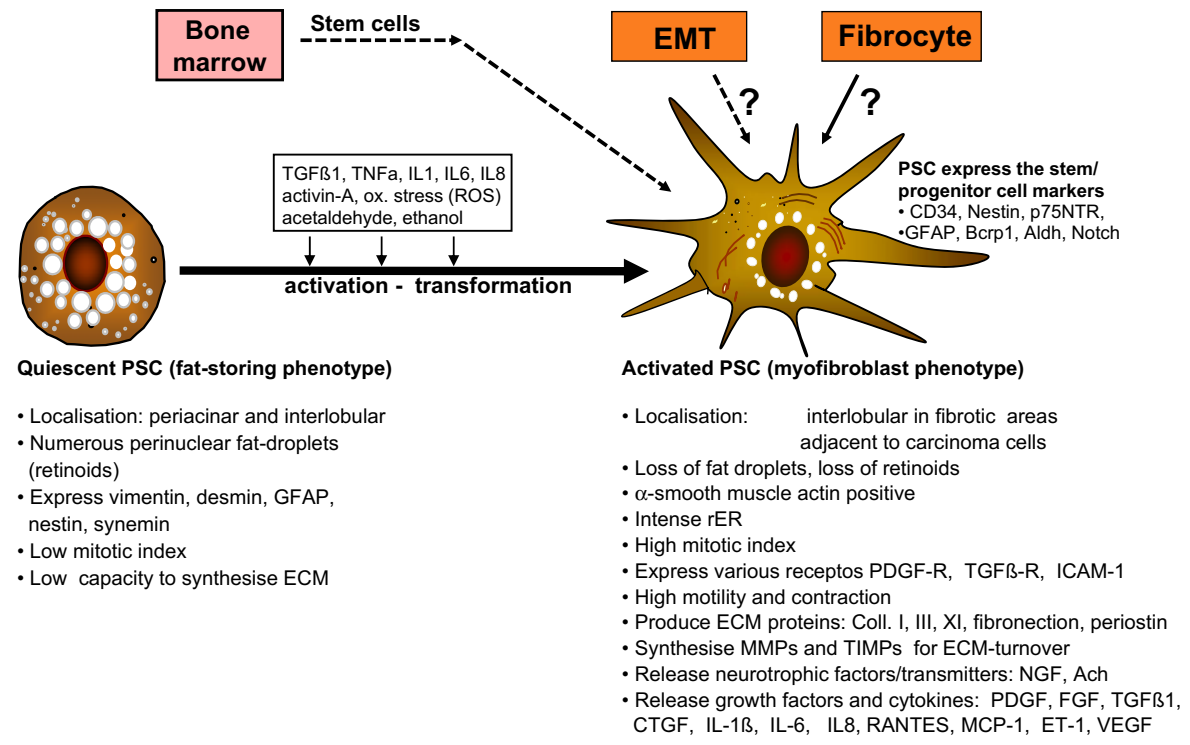

Figure 1 Activation of quiescent stellate cells. Ach, acetylcholine; Aldh, aldehyde dehydrogenase; Bcrp1, breakpoint cluster region pseudogene 1; CD34, bluster of differentiation molecule-34; Coll, collagen; CTGF, connective tissue growth factor; ECM, extracellular matrix; EMT, epithelial mesenchymal transition; ET-1, endothelin-1; FGF, fibroblast-growth-factor; GFAP, glial fibrillar acidic protein; ICAM-1, inter-cellular adhesion molecule 1; IL1, Interleukin-1; IL-1 $\beta$, interleukin-1 beta; IL6, interleukin-6; IL8, interleukin-8; MCP-1, monocyte chemotactic protein 1; MMPs, matrix metalloproteinases; NGF, nerve growth factor; ox. stres, oxidative stress; p75NTR, p75 neurotrophin receptor; PDGF, platelet-derived growth factor; PDGF-R, platelet-derived growth factor receptor; PSC, pancreatic stellate cells; RANTES, regulated upon activation, normal T-cell expressed, and secreted also known as CCL5; rER, rough endoplasmic reticulum; ROS, reactive oxygen species; TGF $\beta 1$, transforming growth factor beta-1; TGF $\beta-\mathrm{R}$, transforming growth factor beta receptor; TIMPs, tissue inhibitor of metalloproteinases; TNF $\alpha$, tumor necrosis factor-alpha; VEGF, vascular endothelial growth factor. early evidence that although expressed in activated PSC, Notch3, secreted frizzled related protein 5 , Wnt4 and Wnt5a are absent in quiescent PSC (C. Kordes, unpublished data); however, there is agreement that these findings need further validation.

\section{PARAMETERS OF PSC ACTIVATION}

There is strong agreement that activation of PSC in culture can be assessed by several functional parameters. However, it is also recognised that the results of studies of PSC activation may be influenced by many different factors including stellate cell purity, culture media used and age of animals harvested or (for humans) donor age and disease (normal pancreas, chronic pancreatitis, pancreatic cancer). While the response of PSC to individual activating factors has been well characterised in vitro, researchers are also mindful that this may not reflect the true in-vivo situation in which: (1) PSC are exposed to a multiplicity of factors at any one time; (2) there is often redundancy of the effects of cytokines; (3) culture conditions may influence ECM composition; and (4) isolated PSC in culture may behave differently from those in situ where they are surrounded by other cell types (figure 2). Once activated, PSC also secrete endogenous factors that can further activate transformed PSC via autocrine pathways.

\section{INTRACELLULAR SIGNALLING PATHWAYS INVOLVED IN STELLATE CELL ACTIVATION/DEACTIVATION}

The intracellular pathways involved in the 'activation' of PSC have also been intensively analysed as the activity of the stroma significantly impacts on the course of pancreatitis and on tumour behaviour. ${ }^{31}$ Therefore, intracellular pathways are considered to be important as potential therapeutic targets to inhibit/revert stellate cell activity. Pertinently, kinases of the mitogen-activated protein kinase family have been implicated in many aspects of PSC activation, but have been linked most consistently to the transduction of mitogenic signals and the stimulation of cytokine/chemokine (eg, MCP-1 and IL-6) production. ${ }^{32}$ The latter effect is transcriptionally mediated by AP1 and nuclear factor kappa B transcription factors. On the other hand, Smad transcription factors are key mediators of the biological effects of transforming growth factor $\beta 1$ in PSC, stimulating transformation to a myofibroblastic phenotype 


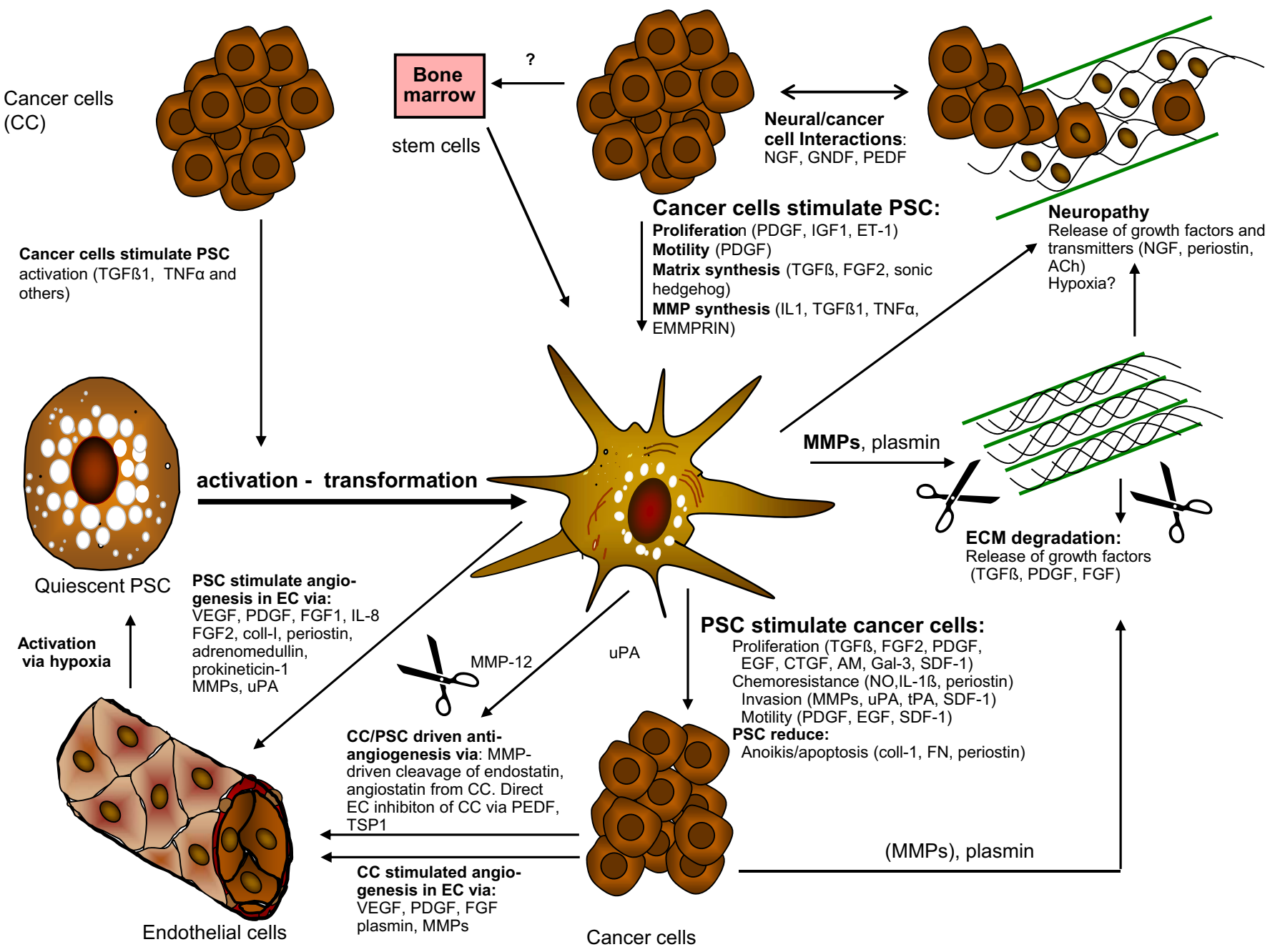

Figure 2 Interactions of pancreatic stellate cells with neighbouring cells in their microenvironment. Ach, acetylcholine; AM, adrenomedullin; CC, cancer cells; Coll, collagen; CTGF, connective tissue growth factor; EC, endothelial cells; ECM, extracellular metrix; EGF, epidermal growth factor; EMMPRIN, extracellular matrix metalloproteinase inducer also known as Basigin or CD147; ET-1, endothelin-1; FGF, fibroblast-growth-factor; GAL-3, galactoside-binding, soluble, 3; GNDF, glial cell line-derived neurotrophic factor; IGF-1, insulin-like growth factor 1; IL1, interleukin-1; IL8, interleukin-8; MMPs, matrix metalloproteinases; NGF, nerve growth factor; NO, nitric oxide; PSC, pancreatic stellate cells; PDGF, platelet-derived growth factor; PEDF, pigment epithelium derived factor; SDF-1, stromal cell-derived factor-1; TGF $\beta 1$, transforming growth factor beta-1; TNF $\alpha$, tumor necrosis factor-alpha; tPA, tissue plasminogen activator; TSP1, thrombospondin 1; uPA, urokinase-type plasminogen activator; VEGF, vascular endothelial growth factor.

and increased ECM synthesis. ${ }^{32}$ However, while several signalling pathways mediating PSC functions have been identified, it is currently not possible to clarify their 'restricted' role as other pathways mediating similar functions are increasingly being identified and the cross-talk between these pathways is also being elucidated.

Signalling pathways that are responsible for PSC inactivation (reversal to a relatively quiescent phenotype) have also been identified in recent years. Inhibition of mitogen-activated protein kinase (ERK, JNK and p38 kinase) signalling in PSC mediates the induction of quiescence in response to retinol and retinoic acid. There is some evidence that the forced expression of PPAR- $\gamma$, C/EBP- $\alpha$, or albumin may also inactivate PSC. ${ }^{33}$ Therefore, modulation of PPAR- $\gamma$ and STAT1, which can be targeted by clinically applicable drugs (thiazolidinediones and interferons, respectively), deserves further investigation.

\section{PSC ARE THE KEY FIBROGENIC CELLS IN THE PANCREAS AND ARE DIFFERENT FROM PFB}

Differentiation between PSC and pancreatic fibroblasts is an important issue given that the outgrowth method of PSC isolation involves the use of fibrotic pancreatic tissue. In contrast to pancreatic fibroblasts (PFB), PSC express $\alpha$-SMA and form dense bodies (microfilaments) like myofibroblastlike cells. ${ }^{4}$ Compared with PSC, PFB are less adherent therefore detach earlier from plastic during trypsinisation. Using this difference, pure cultures of PSC and PFB can be obtained after repeated mild trypsinisation and separate cultivation. ${ }^{34}$ In contrast to PSC, which show a star-like shape (few are also triangular or spindleshaped), cultured PFB are spindle-shaped and smaller. Both cell types are vimentin positive; $20-40 \%$ of PSC are desmin positive and more than $90 \%$ are $\alpha$-SMA positive (positivity increases as cells are kept on plastic). ${ }^{34}$ In contrast, PFB are desmin and $\alpha$-SMA negative. Importantly, PSC produce higher amounts of ECM proteins compared with $\mathrm{PFB}$, and express the scavenger receptor CD36, CCK receptors $1 / 2$ and $\mathrm{ACh}$ receptor while $\mathrm{PFB}$ do not express any of these receptors. ${ }^{34} 35$ 


\section{VARIANCES OF PSC BETWEEN SPECIES, DONORS AND IMPACT OF IMMORTALISATION ON INTERPRETING RESULTS}

There is strong agreement that there are species as well as donor-dependent variances between murine and human PSC. As a result of the limited access to human pancreatic tissue at several institutions, immortalised PSC have been proposed as an alternative model to study pancreatic stroma. Immortalised PSC may be useful for studies of molecular signalling that require manipulation of gene expression and use in in-vivo models that require cells to remain viable for a relatively longer time period. ${ }^{3637}$ However, it is important to note that differences exist between immortalised and primary PSC, and therefore caution must be exercised when extrapolating findings with immortalised PSC to the clinical situation. Of more concern is the observation that immortalised PSC were able (albeit occasionally) to form aggressive anaplastic tumours when injected alone into nude mice (M. Buchholz et al, unpublished data). This raises the possibility that the immortalisation procedure resulted in the preferential selection of malignant subclones. Therefore, there is strong agreement that the results of experiments obtained by using murine or immortalised PSC should ideally be verified using several different primary human PSC cultures to maximise the robustness of the data.

\section{PSC AND PANCREATIC DISEASES}

Compelling evidence has accumulated in recent years to support a major role forPSC in both fibrogenesis and fibrolysis. $^{4}{ }^{38-41}$ During pancreatic injury, PSC are transformed (in response to factors that are now well identified, including oxidant stress, cytokines, growth factors and toxins such as alcohol and its metabolite acetaldehyde) from their quiescent state to an activated myofibroblast-like phenotype that synthesises and secretes excessive amounts of ECM proteins (increased fibrogenesis). Activated PSC are seen in the early phases of alcoholic chronic pancreatitis and in autoimmune pancreatitis. ${ }^{42-44}$ However, it is unclear how fibrogenesis is initiated in the inflamed pancreas. There are currently two concepts. The first concept focuses on the direct activation of PSC by acetaldehyde (the oxidative metabolite of alcohol) and oxidant stress (as shown in vitro and in animal experiments). ${ }^{44}$ The second concept is based on the necrosis-fibrosis sequence as the underlying pathogenic mechanisms of alcoholic chronic pancrea- titis. It is proposed that the initial lesion is autodigestive tissue necrosis, which is followed by inflammation and the induction of the fibrotic reaction. ${ }^{39} 4243$ In stage I with overt tissue injury, PSC are found in close association with macrophages around areas of necrosis. In stage II with extensive cellular fibrosis, PSC are found in the perilobular spaces. In stage III with established dense fibrosis in the perilobular spaces, PSC are conspicuously reduced in number, and in stage IV, when in addition to perilobular fibrosis calculi in the ducts are present, PSC are mainly detected adjacent to duct ulcerations caused by calculi. ${ }^{43}$ There is general agreement that in the early stages (I and II) cessation of alcohol would prevent the formation of organ fibrosis, predominantly through increased apoptosis of activated PSC. ${ }^{39} 42-44$ However, there is currently not enough evidence showing that increasing the fibrolytic activity of PSC reverts organised fibrosis of the pancreas.

The tumour microenvironment is known to be an important contributor to the malignant phenotype. ${ }^{36} 384145-48$ In the pancreas, PSC have been identified within the tumour microenvironment of pancreatic ductal adenocarcinoma. Recent evidence from animal experiments suggests that PSC can promote local tumour growth and metastatic spread and can also increase resistance to chemo and radiation treatment. $^{36} 384145-48$ The vascularity of the tumour is believed to play a role in the aggressive behaviour of pancreatic cancer. Pancreatic ductal adenocarcinoma as well as chronic pancreatitis is characterised by hypoxia and fibrosis. ${ }^{46} 4749$ It remains unclear whether PSC play an overall proangiogenic or anti-angiogenic role in pancreatic fibrosis and cancer. ${ }^{36}{ }^{46-49}$ PSC activity is inversely correlated with vascular density, and inhibition of stellate cell activity results in increased vascularity and the delivery of chemotherapeutic reagents. ${ }^{46} 49$ On the other hand, conditioned media of PSC induce angiogenesis both in vitro and in vivo through the production of vascular endothelial growth factor and non-vascular endothelial growth factor family members. ${ }^{46} 47$ These contradictory findings could be explained by the dynamic rather than on/off responses of PSC. It is likely that PSC may exert different effects on angiogenesis depending on the site (invading front vs dense fibrotic areas) and disease stage (early vs advanced). Pertinently, sonic hedgehog signalling has been implicated in PSC activation and suggested as an important enhancer of the desmoplastic reaction and inhibitor of stromal angiogenesis in pancreatic cancer. $^{49} 50$ However, the downstream targets of hedgehog signalling in PSC remain to be fully elucidated.

\section{TRANSGENIC MOUSE MODELS OF PANCREATIC CANCER AND RE-CREATION OF FIBROTIC TUMOUR MICROENVIRONMENT}

Genetically engineered mouse models of pancreatic cancer, ie, mice with a pancreasspecific activation of oncogenic Kras, have been instrumental in defining the steps from epithelial transformation to invasive tumour growth. ${ }^{5152}$ On a morphological level, these models reflect the presumed stepwise progression from intraepithelial neoplasia to ductal adenocarcinoma. In particular, some of the mouse tumours such as $p 48^{+/ C r e} ; L S L-K R A S^{G 12 D /+}$ and the PDX-1-Cre;LSL-KRAS ${ }^{G 12 D /+}$ are accompanied by a significant desmoplastic response, thus resembling human pancreatic ductal adenocarcinoma. However, it has been unclear for a long time whether a provisional matrix is already present around precursor lesions, whether PSC are part of this matrix and whether desmoplasia is a lesion-specific response and, in particular, whether the excessive matrix has any clinical relevance. With regard to the last point, a recent paper by Erkan et $a l^{\beta 1}$ has shown a close correlation between the extent of $\alpha$-SMA staining and poor outcome, whereas strong collagen deposition correlated with a favourable outcome in pancreatic cancer. In the genetically engineered mouse models of pancreatic ductal adenocarcinoma, the earliest stromal activation and ECM deposition are found around metaplastic/ dysplastic lesions such as tubular complexes and to a lesser extent around PanIN lesions. However, it is not clear whether this stromal activation is a part of carcinogenesis or an epiphenomenon representing an effort to confine the preneoplastic lesions. ${ }^{53}$ In breast and prostate cancers, such a stromal reaction has been shown to precede the actual cancer. However, despite early evidence in pancreatic cancer, ${ }^{53}$ judging by the stromal activation, it is currently not clear whether tubular complexes are an additional precursor lesion in pancreatic ductal adenocarcinoma.

\section{CONCLUSIONS}

Pancreatic fibrosis, which develops as a consequence of chronic inflammation, leads to the loss of functional parenchyma and probably increases the risk of cancer. As the role of PSC in chronic pancreatitis and pancreatic cancer is increasingly 
clarified, it is anticipated that effective approaches to target PSC specifically will be developed. Such therapeutic strategies would be expected to reduce the fibrosis of chronic pancreatitis, thereby retarding the development of exocrine and endocrine insufficiency, and interrupt the interaction of PSC in the stromal reaction with pancreatic cancer cells, thereby inhibiting tumour progression and improving the otherwise dismal prognosis of this disease. Therefore, at present, the field of PSC research is dynamic and wide open, with significant potential for novel discoveries and major breakthroughs that could have a lasting impact on the treatment of patients with pancreatic diseases. The Pancreatic Star Alliance would particularly like to encourage young researchers to enter this exciting area of pancreatology.

\section{FUTURE RESEARCH DIRECTIONS}

1. PSC origin (lineage tracing studies).

2. Functions in health.

3. Interactions with other pancreatic cells (endocrine cells, immune cells, Schwann cells, nerve cells).

4. Role in pancreatic repair/regeneration. 5. Therapeutic targeting.

Figures presented in this article are modified from the figures previously published in Cancers 2010, in which authors hold the copyright and Multidisciplinary Digital Publishing Institute (MDPI) is a licensee.

Funding Funding of the meeting and work by the Pancreatic Star Alliance described in this review is supported by grant fundings to ME, from the DFG (GZ: ER 563/3-1, AOBJ: 577801), from the German Federal Ministry of Education and Research (Bundesministerium für Bildung und Forschung - BMBF -ME, MB, IE, HF, TG, JK, CWM) within the "National Genome Research Network" (NGFN-Plus; 01GS08115) and Stiftung Chirurgie TU München. Part of the meeting costs have been sponsored by Abbott.

\section{Competing interests None.}

Contributors ME appears as the corresponding author. All authors have contibuted equally to the manuscript.

Provenance and peer review Commissioned; externally peer reviewed.

Revised 24 October 2011

Accepted 25 October 2011

Published Online First 24 November 2011

Gut 2012;61:172-178. doi:10.1136/gutjn-2011-301220

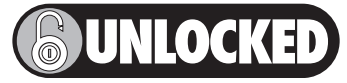

This paper is freely available online under the BMJ Journals unlocked scheme, see http://gut.bmj.com/site/ about/unlocked.xhtml.

\section{REFERENCES}

1. Friedman SL. Hepatic stellate cells: protean, multifunctional, and enigmatic cells of the liver. Physiol Rev 2008:88:125-72.
2. Omary MB, Lugea A, Lowe AW, et al. The pancreatic stellate cell: a star on the rise in pancreatic diseases. J Clin Invest 2007;117:50-9.

3. Watari N, Hotta Y, Mabuchi Y. Morphological studies on a vitamin A-storing cell and its complex with macrophage observed in mouse pancreatic tissues following excess vitamin A administration. Okajimas Folia Anat Jpn 1982;58:837-58.

4. Apte MV, Haber PS, Applegate TL, et al. Periacinar stellate shaped cells in rat pancreas: identification, isolation, and culture. Gut 1998;43:128-33.

5. Bachem MG, Schneider E, Gross H, et al. Identification, culture, and characterization of pancreatic stellate cells in rats and humans. Gastroenterology 1998;115:421-32.

6. Wake K. Perisinusoidal stellate cells (fat-storing cells, interstitial cells, lipocytes), their related structure in and around the liver sinusoids, and vitamin A-storing cells in extrahepatic organs. Int Rev Cytol 1980;66:303-53.

7. Pinzani M, Failli $P$, Ruocco $C$, et al. Fat-storing cells as liver-specific pericytes. Spatial dynamics of agonist-stimulated intracellular calcium transients. J Clin Invest 1992;90:642-6.

8. Asahina K, Tsai SY, Li P, et al. Mesenchymal origin of hepatic stellate cells, submesothelial cells, and perivascular mesenchymal cells during mouse liver development. Hepatology 2009;49:998-1011.

9. Enzan H, Himeno $\mathrm{H}$, Hiroi $\mathrm{M}$, et al. Development of hepatic sinusoidal structure with special reference to the Ito cells. Microsc Res Tech 1997;39:336-49.

10. Cassiman D, Barlow A, Vander Borght S, et al. Hepatic stellate cells do not derive from the neural crest. J Hepatol 2006;44:1098-104.

11. Kiassov AP, Van Eyken P, van Pelt JF, et al. Desmin expressing nonhematopoietic liver cells during rat liver development: an immunohistochemical and morphometric study. Differentiation 1995;59:253-8.

12. Vassy J, Rigaut JP, Briane D, et al. Confocal microscopy immunofluorescence localization of desmin and other intermediate filament proteins in fetal rat livers. Hepatology 1993;17:293-300.

13. Cassiman D, Roskams T. Beauty is in the eye of the beholder: emerging concepts and pitfalls in hepatic stellate cell research. J Hepatol 2002;37:527-35.

14. Friedman SL. Molecular regulation of hepatic fibrosis, an integrated cellular response to tissue injury. J Biol Chem 2000;275:2247-50.

15. Niki T, Pekny M, Hellemans K, et al. Class VI intermediate filament protein nestin is induced during activation of rat hepatic stellate cells. Hepatology 1999;29:520-7.

16. Buchholz M, Kestler HA, Holzmann K, et al. Transcriptome analysis of human hepatic and pancreatic stellate cells: organ-specific variations of a common transcriptional phenotype. $\mathrm{J} \mathrm{Mol} \mathrm{Med}$ 2005; 83:795-805

17. Erkan M, Weis N, Pan Z, et al. Organ-, inflammationand cancer specific transcriptional fingerprints of pancreatic and hepatic stellate cells. Mol Cancer 2010;9:88.

18. Asahina K, Zhou B, Pu WT, et al. Septum transversum-derived mesothelium gives rise to hepatic stellate cells and perivascular mesenchymal cells in developing mouse liver. Hepatology 2011; 53:983-95

19. Higashiyama R, Moro T, Nakao S, et al. Negligible contribution of bone marrow-derived cells to collagen production during hepatic fibrogenesis in mice. Gastroenterology 2009;137:1459-66 e1.

20. Russo FP, Alison MR, Bigger BW, et al. The bone marrow functionally contributes to liver fibrosis. Gastroenterology 2006;130:1807-21.

21. Sparmann G, Kruse ML, Hofmeister-Mielke N, et al. Bone marrow-derived pancreatic stellate cells in rats. Cell Res 2010;20:288-98.
22. Watanabe T, Masamune A, Kikuta K, et al. Bone marrow contributes to the population of pancreatic stellate cells in mice. Am J Physiol Gastrointest Liver Physiol 2009;297:G1138-46.

23. Pinzani M. Epithelial-mesenchymal transition in chronic liver disease: fibrogenesis or escape from death? J Hepatol 2011;55:459-65.

24. Kordes C, Sawitza I, Haussinger D. Hepatic and pancreatic stellate cells in focus. Biol Chem 2009;390:1003-12.

25. Sawitza I, Kordes C, Reister S, et al. The niche of stellate cells within rat liver. Hepatology 2009;50:1617-24.

26. Roskams T. Relationships among stellate cell activation, progenitor cells, and hepatic regeneration. Clin Liver Dis 2008;12:853-60; ix.

27. Mato $\mathbf{E}$, Lucas M, Petriz J, et al. Identification of a pancreatic stellate cell population with properties of progenitor cells: new role for stellate cells in the pancreas. Biochem J 2009;421:181-91.

28. Vonlaufen A, Phillips PA, Yang L, et al. Isolation of quiescent human pancreatic stellate cells: a promising in vitro tool for studies of human pancreatic stellate cell biology. Pancreatology 2010;10:434-43.

29. Haag C, Stadel D, Zhou S, et al. Identification of c-FLIP(L) and c-FLIP(S) as critical regulators of death receptor-induced apoptosis in pancreatic cancer cells. Gut 2011;60:225-37.

30. Krizhanovsky V, Yon M, Dickins RA, et al Senescence of activated stellate cells limits liver fibrosis. Cell 2008;134:657-67.

31. Erkan M, Michalski CW, Rieder S, et al. The activated stroma index is a novel and independent prognostic marker in pancreatic ductal adenocarcinoma. Clin Gastroenterol Hepatol 2008;6:1155-61.

32. Masamune A, Shimosegawa T. Signal transduction in pancreatic stellate cells. J Gastroenterol 2009;44:249-60.

33. $\operatorname{Kim} \mathbf{N}, Y_{00} \mathbf{W}$, Lee J, et al. Formation of vitamin A lipid droplets in pancreatic stellate cells requires albumin. Gut 2009;58:1382-90.

34. Bachem MG, Schmid-Kotsas A, Siech M, et al. Pancreatic stellate cells and their role in fibrogenesis. In: Johnson CD, Imrie CW, eds. Pancreatic Disease, Basic Science and Clinical Management. London: Springer-Verlag, 2004:226-39.

35. Phillips PA, Yang L, Shulkes A, et al. Pancreatic stellate cells produce acetylcholine and may play a role in pancreatic exocrine secretion. Proc Natl Acad Sci U S A 2010;107:17397-402.

36. Hwang RF, Moore T, Arumugam T, et al. Cancerassociated stromal fibroblasts promote pancreatic tumor progression. Cancer Res 2008;68:918-26.

37. Jaster R, Lichte P, Fitzner B, et al. Peroxisome proliferator-activated receptor gamma overexpression inhibits pro-fibrogenic activities of immortalised rat pancreatic stellate cells. J Cell Mol Med 2005;9:670-82

38. Bachem MG, Schunemann M, Ramadani M, et al. Pancreatic carcinoma cells induce fibrosis by stimulating proliferation and matrix synthesis of stellate cells. Gastroenterology 2005;128:907-21.

39. Kloppel G, Detlefsen S, Feyerabend B. Fibrosis of the pancreas: the initial tissue damage and the resulting pattern. Virchows Arch 2004;445:1-8.

40. Phillips PA, McCarroll JA, Park S, et al. Rat pancreatic stellate cells secrete matrix metalloproteinases: implications for extracellular matrix turnover. Gut 2003:52:275-82.

41. Vonlaufen A, Joshi S, Ou C, et al. Pancreatic stellate cells: partners in crime with pancreatic cancer cells. Cancer Res 2008;68:2085-93.

42. Ammann RW, Heitz PU, Kloppel G. Course of alcoholic chronic pancreatitis: a prospective clinicomorphological long-term study. Gastroenterology 1996;111:224-31. 
43. Detlefsen S, Sipos B, Feyerabend B, et al. Fibrogenesis in alcoholic chronic pancreatitis: the role of tissue necrosis, macrophages, myofibroblasts and cytokines. Mod Pathol 2006;19:1019-26.

44. Vonlaufen A, Phillips PA, Xu Z, et al. Withdrawal of alcohol promotes regression while continued alcohol intake promotes persistence of LPS-induced pancreatic injury in alcohol-fed rats. Gut 2011;60:238-46.

45. Erkan M, Kleeff J, Gorbachevski A, et al. Periostin creates a tumor-supportive microenvironment in the pancreas by sustaining fibrogenic stellate cell activity. Gastroenterology 2007;132:1447-64.

46. Erkan M, Reiser-Erkan C, Michalski CW, et al. Cancer-stellate cell interactions perpetuate the hypoxia-fibrosis cycle in pancreatic ductal adenocarcinoma. Neoplasia 2009;11:497-508.

47. Masamune A, Kikuta K, Watanabe T, et al. Hypoxia stimulates pancreatic stellate cells to induce fibrosis and angiogenesis in pancreatic cancer. $A m \mathrm{~J}$ Physiol Gastrointest Liver Physiol 2008;295 G709-17.

48. Xu Z Z Vonlaufen A, Phillips PA, et al. Role of pancreatic stellate cells in pancreatic cancer metastasis. Am J Pathol 2010;177:2585-96.

49. Olive KP, Jacobetz MA, Davidson CJ, et al. Inhibition of Hedgehog signaling enhances delivery of chemotherapy in a mouse model of pancreatic cancer. Science 2009:324:1457-61.
50. Bailey JM, Swanson BJ, Hamada T, et al. Sonic hedgehog promotes desmoplasia in pancreatic cancer. Clin Cancer Res 2008;14:5995-6004.

51. Hingorani SR, Petricoin EF, Maitra A, et al. Preinvasive and invasive ductal pancreatic cancer and its early detection in the mouse. Cancer Cell 2003;4:437-50.

52. Ji B, Tsou L, Wang $\mathrm{H}$, et al. Ras activity levels control the development of pancreatic diseases. Gastroenterology 2009;137:1072-82; 82 e1-6.

53. Esposito I, Seiler C, Bergmann F, et al. Hypothetical progression model of pancreatic cancer with origin in the centroacinar-acinar compartment. Pancreas 2007;35:212-17.

\section{SAVE TIME AND KEEP INFORMED} SCAN. SIGN UP. ETOC.

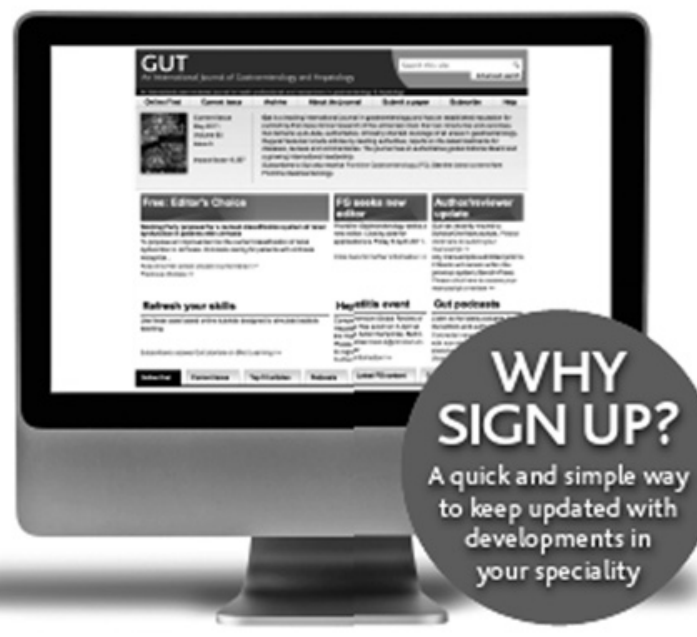

gut.bmj.com
Utilise our Quick Response code (QR) to sign up for our electronic table of contents (eTOC) alert.

To make this simple you can sign up now via your Smartphone.

\section{FOLLOWTHESE}

\section{THREE EASY STEPS:}

1. Downbad a free $Q R$ reader from your handset's app store

2. Hold your Smartphone over the $Q R$ code

3. You will then be forwarded to the eTCC sign up page

To find out more about $Q R$ codes visit

group.bmj.com/products/journals/qr-codes 